\title{
HOXA-AS2 Promotes Proliferation and Induces Epithelial-Mesenchymal Transition via the miR-520c-3p/GPC3 Axis in Hepatocellular Carcinoma
}

\author{
Ying Zhang ${ }^{\mathrm{a}}$ Jianliang $X \mathrm{u}^{\mathrm{b}} \quad$ Shaoquan Zhang ${ }^{\mathrm{a}} \quad$ Jun $^{\mathrm{A}} \mathrm{n}^{\mathrm{c}} \quad$ Jin Zhang ${ }^{\mathrm{d}}$ \\ Jianglong Huang ${ }^{e}$ Yi Jin ${ }^{f}$
}

\begin{abstract}
aDepartment of Infectious Diseases, the Third Affiliated Hospital, Sun Yat-Sen University, Guangzhou, bDepartment of Hepatobiliary Surgery, the Third Affiliated Hospital, Sun Yat-Sen University, Guangzhou, 'Department of Cardiothoracic Surgery, the third Affiliated Hospital of Sun Yat-Sen University, Guangzhou, dDepartment of Thoracic Surgery, the Second Affiliated Hospital of Xi'an Jiaotong University, Xi'an, 'Department of Gastrointestinal Surgery, the third Affiliated Hospital of Sun Yat-Sen University, Guangzhou, 'Department of Pathology, Guangdong Provincial Key Laboratory of Liver Disease Research, the Third Affiliated Hospital, Sun Yat-Sen University, Guangzhou, China
\end{abstract}

\section{Key Words}

Hoxa-AS2 $・$ LncRNA $\cdot H C C \cdot$ miR-520c-3p • EMT • Therapeutic target

\begin{abstract}
Background/Aims: Previous studies have demonstrated that long non-coding RNAs (IncRNAs) may play critical roles in cancer biology, including Hepatocellular carcinoma (HCC). The HOXA cluster antisense RNA2 (HOXA-AS2) IncRNA plays an important role in carcinogenesis, however, the underlying role of HOXA-AS2 in HCC remains unknown. The present study examined the effects of HOXA-AS2 on the progression of HCC, and explored the underlying molecular mechanisms. Methods: Quantitative real-time PCR was used to detect HOXA-AS2 expression in HCC tissues and cell lines. Furthermore, the effects of HOXA-AS2 silencing and overexpression on cell proliferation, cell cycle, apoptosis, migration, and invasion were assessed in HCC in vitro and in vivo. Furthermore, bioinformatics online programs predicted and luciferase reporter assay were used to validate the association of HOXA-AS2 and miR$520 c-3 p$ in HCC cells. Results: We observed that HOXA-AS2 was up-regulated in HCC tissues and cell lines. In vitro experiments revealed that HOXA-AS2 knockdown significantly inhibited HCC cells proliferation by causing G1 arrest and promoting apoptosis, whereas HOXA-AS2 overexpression promoted cell growth. Further functional assays indicated that HOXA-AS2 significantly promoted HCC cell migration and invasion by promoting EMT. Bioinformatics online programs predicted that HOXA-AS2 sponge miR-520c-3p at 3'-UTR with complementary binding sites, which was validated using luciferase reporter assay. HOXA-AS2 could negatively regulate the expression of miR-520c-3p in HCC cells. MiR-520c-3p was down-regulated and

Y. Zhang, J. Xu and S. Zhang contributed equally to this work.

\footnotetext{
Yi Jin Department of Pathology, Third Affiliated Hospital, Sun Yat-Sen Univ., 600 Tianhe Road,

and Jianglong Huang Guangzhou; Department of Gastrointestinal Surgery, the third Affiliated Hospital of Sun Yat-Sen University, Guangzhou (China), E-Mail dryijin16@163.com; drhongj16@163.com
}

\section{KARGER}




\section{Cellular Physiology Cell Physiol Biochem 2018;50:2124-2138 \begin{tabular}{ll|l} 
and Biochemistry & $\begin{array}{l}\text { DOl: 10.1159/000495056 } \\
\text { Published online: 10 November } 2018\end{array}$ & $\begin{array}{l}\text { O } 2018 \text { The Author(s). Published by S. Karger AG, Basel } \\
\text { www.karger.com/cpb }\end{array}$ \\
\cline { 2 - 3 }
\end{tabular} \\ Zhang et al.: HOXA-AS2 and HCC}

inversely correlated with HOXA-AS2 expression in HCC tissues. miR-520c-3p suppressed cell proliferation, invasion and migration in HCC cells, and enforced expression of miR-520c-3p attenuated the oncogenic effects of HOXA-AS2 in HCC cells. By bioinformatic analysis and dual-luciferase reporter assay, we found that miR-223-3p directly targeted the 3'-untranslated region (UTR) of Glypican-3 (GPC3), one of the key players in HCC. GPC3 was up-regulated in $\mathrm{HCC}$ tissues, and was negatively correlated with miR-520c-3p expression and positively correlated with HOXA-AS2 expression. Conclusion: In summary, our results suggested that the HOXA-AS2/miR-520c-3p/GPC3 axis may play an important role in the regulation of PTC progression, which could serve as a biomarker and therapeutic target for HCC.

(C) 2018 The Author(s)

Published by S. Karger AG, Basel

\section{Introduction}

Hepatocellular carcinoma (HCC) is one of the most malignant tumors, with a low 5-year survival rate $[1,2]$. Hepatic resection remains a potentially curative treatment for HCC, but the prognosis for HCC has not significantly improved [3]. Tumour metastasis is one of the major causes of the high mortality [4]. Although great progress has been achieved in the study of the HCC in recent decades, the molecular mechanism underlying HCC metastasis still remains elusive. Thus, there is an urgent need to develop new strategies to HCC treatment.

Long noncoding RNA (lncRNA) is a type of noncoding RNA greater than 200 nucleotides in length [5,6]. Recent studies showed that lncRNA can act as oncogenes or tumor suppressors in malignant tumors, suggesting that the aberrant expression of lncRNAs is associated with tumorigenesis of many types of cancers [7,8]. Abnormal lncRNA expression and its association with various important clinicopathological parameters have been reported in HCC $[9,10]$. Recently, increasing evidence has shown that HOXA cluster antisense RNA 2 (HOXA-AS2), a lincRNA located between and antisense to the human HOXA3 and HOXA4 genes, promote several tumorigenic features including survival, proliferation and invasion [11]. However, the roles of IncRNA HOXA-AS2 on HCC progression and the related mechanisms are still unclear. In this study, we explored the role of HOXA-AS2 in the regulation of proliferation, migration and invasion of HCC.

Many studies have shown that lncRNAs can serve as a competing endogenous RNAs (ceRNAs) to regulate microRNAs (miRNAs) $[12,13]$. CeRNAs play a post-transcriptional regulatory role in miRNA distribution on their targets. miRNAs are a class of 21-25 nucleotide small non-coding RNA with no protein-coding potential. miRNAs have crucial roles in the regulation of gene expression through binding to the 3'-UTR of the target genes [14]. Recent studies have also demonstrated that the dysregulation of miRNAs is involved in a wide range of human cancers, including HCC. More and more studies had found that miR-520c-3p influences the biological progression of various cancer cells [15]. Recent study has demonstrated that miR-520c-3p negatively regulates epithelial-mesenchymal transition (EMT) by targeting IL-8 to suppress the invasion and migration of breast cancer [16]. In addition, miR-520c-3p could target the 3'-UTR of S100A4 and inhibits its expression and its mediated migration and invasion of colorectal cancer [17].

The present study aimed to explore the ceRNA mechanism of HOXA-AS2 though miR$520 c-3 p$ and revealed the functional relevance of miR-520c-3p and HOXA-AS2 in HCC. In this study, we found that upregulation of HOXA-AS2 in HCC tissues and cells were associated with tumor progression, and HOXA-AS2 could function as a ceRNA to regulate the expression of Glypican-3 (GPC3) by competing for miR-520c-3p binding. 


\section{Cellular Physiology Cell Physiol Biochem 2018;50:2124-2138 \begin{tabular}{l|l|l} 
and Biochemistry Published online: 10 November 2018 & $\begin{array}{l}\text { (c) } 2018 \text { The Author(s). Published by S. Karger AG, Basel } \\
\text { www.karger.com/cpb }\end{array}$ \\
\hline
\end{tabular} \\ Zhang et al.: HOXA-AS2 and HCC}

\section{Materials and Methods}

\section{Human tissue specimens}

A total of 58 samples of HCC tissues and paired adjacent noncancerous liver tissues were obtained from patients who underwent surgery at Department of Hepatobiliary Surgery, the Third Affiliated Hospital, Sun Yat-Sen University between 2010 and 2016. All the patients were pathologically confirmed and the tissues were collected immediately after they were obtained during the surgical operation, and then stored at $-80^{\circ} \mathrm{C}$ to prevent RNA loss. No systemic or local treatment had been received before operation. Both nontumor and tumor tissues were histologically confirmed. Informed consent was obtained from each patient and was approved by the Institute Research Ethics Committee at Sun Yat-Sen University.

\section{Cell culture}

HCC cell lines MHCC97L, Huh7, HepG2, HCCLM3, SMMC-7721, MHCC97H and normal liver cell lines HL-7702 were from the tumor cell bank of Chinese Academy of Sciences. All the cell lines were grown in Dulbecco's modified eagle medium supplemented with $10 \%$ fetal bovine serum, $100 \mathrm{Ag} / \mathrm{AL}$ streptomycin, and $100 \mathrm{Ag} / \mathrm{AL}$ penicillin ( $\mathrm{pH} 7.2-7.4$ ) in a humidified incubator containing $5 \% \mathrm{CO}_{2}$ at $37{ }^{\circ} \mathrm{C}$.

\section{RNA extraction and $q R T-P C R$ assays}

Total RNA was extracted from tissues or cultured cells using TRIZOL reagent (Invitrogen). For qRTPCR, RNA was reverse transcribed to cDNA by using a Reverse Transcription Kit (Takara, Dalian, China). Real-time PCR analyses were performed with SYBR Premix Ex Taq (Takara, Dalian China). Results were normalized to the expression of GAPDH. The sequence of the primers were as following: HOXA-AS2 (Forward: 5'-CCCGTAGGAAGAACCGATGA-3', Reverse: 5'-TTTAGGCCTTCGCAGACAGC-3') and GAPDH (Forward: 5'-GGGAGCCAAAAGGGTCAT-3', Reverse: 5'-GAGTCCTTCCACGATACCAA-3'). The qRT-PCR assays were conducted on an ABI 7500, and data collected with this instrument. Our qRT-PCR results were analyzed and expressed relative to threshold cycle (CT) values, and then converted to fold changes.

\section{Transfection}

To perform effective lentivirus-mediated suppression of HOXA-AS2 in HCC cells, the following HOXAAS2 shRNA and scrambled control shRNA were inserted into the pLVX-tdTomato-Puro vector (Biowit, Shenzhen, China): HOXA-AS2 shRNA\#1 forward, 5'- GAGUUCAGCUCAAGUUGAACAUACA-3' and reverse, 5'UGUAUGUUCAACUUGAGCUGAACUC-3'; shRNA\#2 forward, 5'- AAACCUUGUAGAUAGCUUGAGCUGG-3' and reverse, 5' - CCAGCUCAAGCUAUCUACAAGGUUU-3'; shRNA\#3 forward, 5'-CAAGCUUGACAAGUUCAGCUCAA-3' and reverse, 5'- UUGAGCUGAACUCUUGUCAAGCUUG-3'; The full-length complementary DNA of HOXA-AS2 was synthesized by Realgene (Nanjing, China) and subcloned into the pcDNA3.1 (+) vector (Invitrogen) according to the manufacturer's instructions. At $48 \mathrm{~h}$ post-transfection, cells were harvested for qRT-PCR analysis.

\section{CCK-8 cell viability assay}

After transfection, the cells mixed with $10 \mathrm{ml}$ of CCK-8 solutions per well and incubated for further $1 \mathrm{~h}$ at $37^{\circ} \mathrm{C}$. The amount of formazan dye generated by cellular dehydrogenase activity was measured for absorbance at $450 \mathrm{~nm}$ by a microplate reader (Molecular Devices, Sunnyvale, CA, USA). The optical density values of each well represented the survival/proliferation of HCC cells.

\section{Colony formation assays}

For the colony formation assay, 500 cells were placed in six-well plates and maintained in complete medium for 2 weeks. Colonies were fixed with methanol, stained with $0.1 \%$ crystal violet and counted.

\section{Cell migration andinvasion assays}

A total of $5 \times 10^{4}$ transfected cells were resuspended in $200 \mu \mathrm{l}$ of serum-free medium and placed in the upper compartment of a Transwell chamber (Corning; 24 -well insert, pore size: $8 \mu \mathrm{m}$ ). The lower chamber was filled with $15 \%$ foetal bovine serum as a chemoattractant and incubated for $48 \mathrm{~h}$ for the migration assay and $72 \mathrm{~h}$ for the invasion assay. For the invasion assay, the inserts were previously coated with extracellular matrix gel (BD Biosciences, Sparks, MD, USA). At the end of the experiments, the cells on the upper surface 


\section{Cellular Physiology Cell Physiol Biochem 2018;50:2124-2138

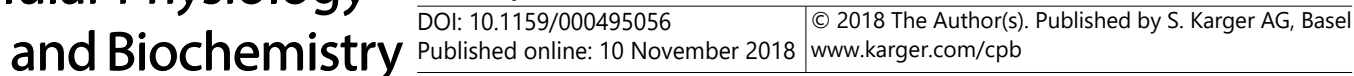 \\ Zhang et al.: HOXA-AS2 and HCC}

of the membrane were removed, and the cells on the lower surface were fixed and stained with $0.1 \%$ crystal violet. Five visual fields of each insert were randomly chosen and counted under a light microscope.

\section{Western blotting}

Total protein was extracted from cells using RIPA lysis buffer (Beyotime, Jiangsu, China). Samples of cell lysate protein were separated by sodium dodecyl sulfate polyacrylamide gel electrophoresis (SDS-PAGE) and transferred onto polyvinylidene fluoride (PVDF) membranes. After blocking with 5\% non-fat milk in TBS-T, proteins were then labelled with anti-E-cadherin, anti-N-cadherin, anti-vimentin 1:1000; Abcam Inc., Cambridge, MA, USA) and anti-GAPDH (Cell Signaling Technology, Beverly, MA, USA) primary and secondary antibodies and detected with Image Acquisition using Image Quant ${ }^{\mathrm{TM}}$ LAS 4000 (GE Healthcare Life Sciences, Michigan, USA).

\section{Animal experiments}

The purchase of 4 week old female BALB/c nude mice was made from the Shanghai Experimental Animal Center of Chinese Academy of Sciences (Shanghai, China). All of the experimental procedures were carried out, subsequent to the approval received from the Animal Care and Experiment Committee of Third Affiliated Hospital, Sun Yat-Sen University. The HCCLM3 cells were steadily transfected with HOXA-AS2 shRNA as well as negative control (GenePharma), in addition to subcutaneous injection into the posterior flank of BALB/C nude mice (six mice in a group). Weekly measurements of tumour developments were taken. Moreover, 5 weeks following the injection, the mice were euthanized and tumour weights were detected.

\section{Luciferase reporter assays}

StarBase v2.0 (http://starbase.sysu.edu.cn/) was used to predict the binding sites between LncRNAHOXA-AS2 and miR-520c-3p. The luciferase reporter assays were carried out with the help of the Dualluciferase Reporter Assay System (Promega, Madison, WI, USA). The wide-type HOXA-AS2 or mutant HOXAAS2 that had the predicted.

miR-520c-3p binding site was established and integrated into a pmir-GLO Dual-luciferase vector to form the pmirGLO-HOXA-AS2-wild type (HOXA-AS2-wt) or pmirGLO- HOXA-AS2-mutant (HOXA-AS2-mut) reporter vector. Cotransfection of HOXA-AS2-wt or HOXA-AS2-mut was carried out with miR-520c-3p mimics or negative control into HCC cells with the use of Lipofectamine 2000. Subsequent to transfection for a period of 48 hours, the luciferase activities were measured in accordance with the guidelines of the manufacturer. In the same manner, pmirGLO-GPC3-wild type (GPC3-wt) or pmirGLO-GPC3-mutant (GPC3mut) were constructed, together with cotransfecting with miR-520c-3p mimics or negative control into HEK293T cells. 48 hours following the transfection, the relative luciferase activities were detected.

\section{Immunohistochemistry}

For each patient sample, three paraffin sections of $5 \mu \mathrm{m}$ were prepared, one for hematoxylin and eosin (HE) staining and the other two for immunohistochemical staining. PBS instead of primary antibodies was used for negative control, and the breast cancer tissue was used for positive control. Sections were dewaxed using xylene, followed by hydration with ethanol solutions and addition of EDTA for antigen retrieval. Later, sections were blocked with normal goat serum for $30 \mathrm{~min}$ to eliminate non-specific binding. Sections were incubated with primary antibody against GPC3 (Abcam, Cambridge, UK). Sections were then incubated with biotin-labeled secondary antibodies for $30 \mathrm{~min}$ at room temperature, followed by staining with diaminobenzidine (DAB). Finally, the sections were counterstained with hematoxylin. The result of staining was determined by two doctors who did not know the clinical condition of patients. The proportions of positive cells of $0,1-5,6-25,26-75$, and 76-100 \% were assigned with scores of $0,1,2,3$, and 4, respectively. Scores of $0-2$ were considered as negative expression, and scores of 3-4 were considered as positive expression.

\section{Statistical analysis}

The presentation of the data has been made as the mean \pm SD from at least three sovereign experiments. Statistical analyses were carried out with the use of SPSS 18.0 software (SPSS, Chicago, IL, USA), together with generating the graphs using GraphPad Prism 6.0 (GraphPad Prism, San Diego, CA, USA). The relationship between HOXA-AS2 expression and clinical characteristics of HCC patients was evaluated with 


\section{Cellular Physiology Cell Physiol Biochem 2018;50:2124-2138

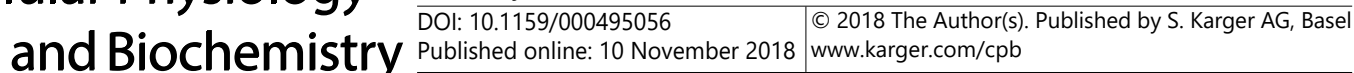 \\ Zhang et al.: HOXA-AS2 and HCC}

the application of the chi-squared test. Analyses were carried out for the dissimilarities between groups through the application of Student's $t$ test, one-way ANOVA analysis and Pearson's correlation analysis. $P<$ 0.05 was considered statistically significant.

\section{Results}

LncRNA-HOXA-AS2 was up-regulated in human HCC tissues and cell lines

Firstly, we analyzed the expression levels of HOXA-AS2 in 58 paired human primary HCC tissues and their corresponding nontumourous liver tissues by qRT-PCR analysis, and found that HOXA-AS2 expression levels were upregulated in tumor tissues compared with normal tissues $\left({ }^{* *} P<0.01\right.$; Fig. 1A). Next, we found that high expression of HOXA-AS2 was corrected with distant metastasis $\left({ }^{* *} P<0.01\right.$; Fig. 1B). In addition, the relationships between HOXAAS2 expression and clinical characteristics in 58 patients with HCC were analyzed, and we found that expression was significantly associated with distant metastasis $(P=0.025)$ and TNM stage $(P=0.020)$ but not related to gender and age (Table 1$)$. These results suggested that HOXA-AS2 might be an oncogene in HCC.

To investigate whether the expression of HOXA-AS2 was altered in HCC, we measured the expression of HOXA-AS2 in a panel of HCC cell lines by qRT-PCR. Our results showed that HOXA-AS2 was increased in all of the 6 analysed HCC cell lines compared with the normal liver cell lines HL-7702. Furthermore, HCCLM3 and MHCC97H cells (high metastatic potential) showed the higher expression of HOXA-AS2, in relation to Huh7 and SMMC7721 cells (low metastatic potential) $(P<0.01$; Fig. $1 \mathrm{C})$.

To analyze the role of HOXA-AS2 in HCC, HCCLM3 and MHCC97H cells were stably transfected with HOXA-AS2 shRNAs (respectively sh HOXA-AS2 \#1, shHOXA-AS2 \#2, or shHOXA-AS2 \#3) or empty vectors (sh-NC). We detected HOXA-AS2 expression at $48 \mathrm{~h}$ posttransfection by qRT-PCR analysis to analyze knockdown efficiency and revealed that shHOXAAS2 \#2 had higher efficiency of interference than shHOXA-AS2 \#1 and \#3 group (Fig. 1D), so we chose shHOXA-AS2 \#2 subsequently for the following experiments. Meanwhile, we induced ectopic overexpression of HOXA-AS2 by transfecting Huh7 cell lines with pcDNAHOXA-AS2 expression vector (Fig. 1E).

\section{HOXA-AS2 promotes HCC cell proliferation in vitro}

CCK8 assays showed that silencing of HOXA-AS2 expression significantly inhibited cell proliferation of HCCLM3 and MHCC97H cells compared with the respective controls (Fig. $1 \mathrm{~F}-\mathrm{G})$. Whereas, stimulated HOXAAS2 expression promoted Huh7 cell growth (Fig. 1H). Furthermore, colony-formation assays also indicated that clonogenic survival was significantly decreased following silencing of HOXA-AS2 in HCCLM3 and MHCC97H cells, but markedly increased in Huh7 cells after overexpression of HOXA-AS2 $(P<$ 0.05, Fig. 1I).

Next, flow cytometric analysis was performed further to evaluate whether HOXA-AS2 could impact proliferation of HCC cells by altering cell-cycle progression or apoptosis. Compared with control cells, knockdown of HOXA-AS2 caused cell cycle arrest in G0/G1 phase 48

Table 1. Correlation between HOXA-AS2 expression and clinicopathologic characteristics of HCC patients

\begin{tabular}{llll}
\hline Characteristics & $\begin{array}{l}\text { HOXA-AS2 } \\
\text { Low } \\
(\mathrm{n}=20)\end{array}$ & $\begin{array}{l}\text { expression } \\
\text { High } \\
(\mathrm{n}=38)\end{array}$ & $P$ \\
\hline Age, $\mathbf{y}$ & & & \\
$\geq 51$ & 12 & 24 & 0.642 \\
$\begin{array}{l}\text { Gender } \\
\text { Female }\end{array}$ & 11 & 22 & 0.820 \\
$\begin{array}{l}\text { Serum AFP } \\
\geq 20\end{array}$ & 11 & 20 & 0.235 \\
$\begin{array}{l}\text { Tumor size } \\
\geq 5.0 \text { cm } \\
\text { TNM stage }\end{array}$ & 9 & 18 & 0.235 \\
$\begin{array}{l}\text { III/IV } \\
\text { Distant metastasis }\end{array}$ & 5 & & \\
Yes & 6 & 23 & 0.020 \\
\hline
\end{tabular}




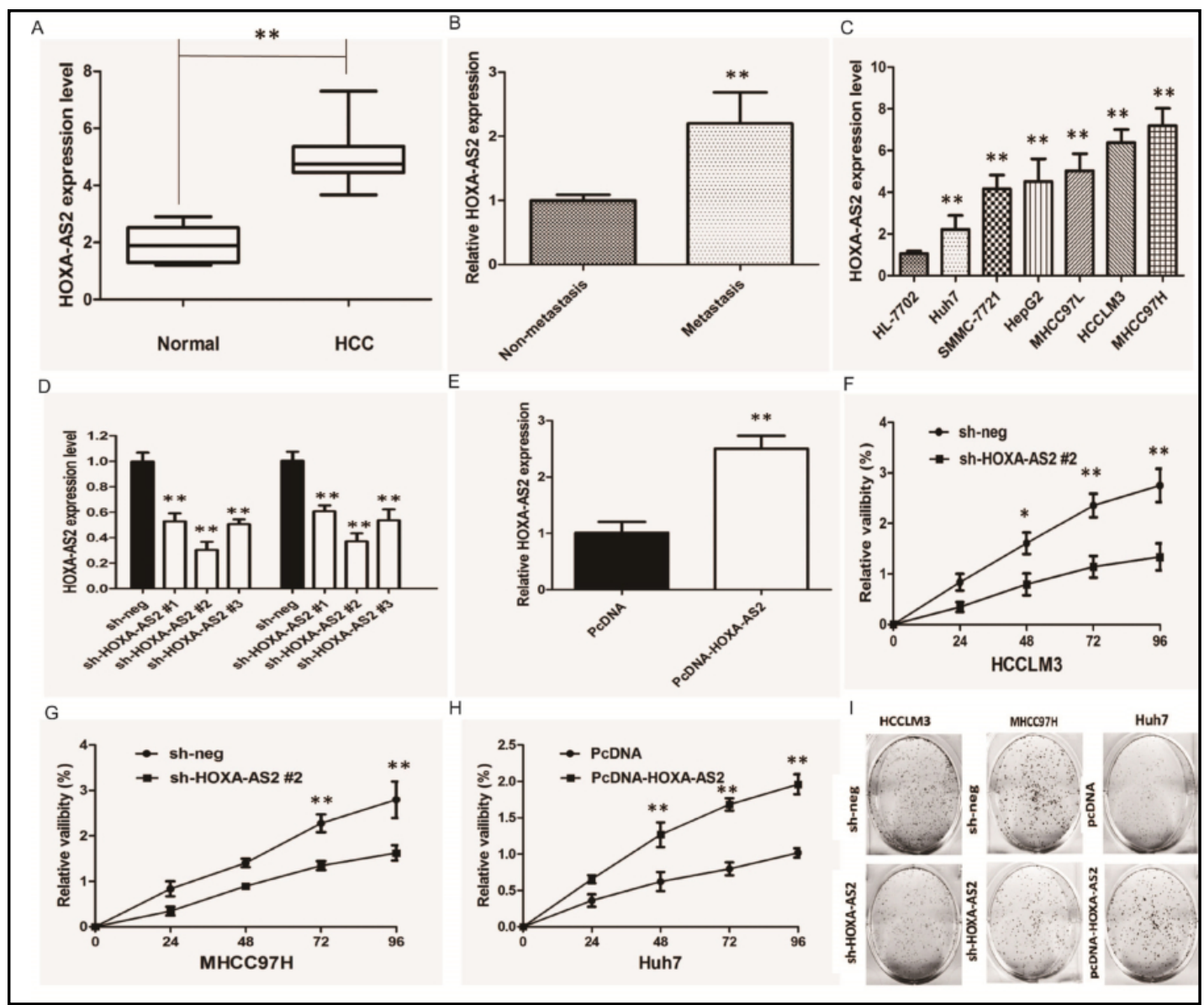

Fig. 1. A: qRT-PCR showing expression level of HOXA-AS2 in HCC tissues and adjacent noncancerous tissues; B: qRT-PCR showing expression level of HOXA-AS2 in metastasis tissues; C: qRT-PCR showing expression level of HOXA-AS2 in HCC cell lines; D: HOXA-AS2 shRNAs was used to enhance efficiency of HOXA-AS2 knockdown in HCCLM3 and MHCC97H cells; E: ectopic overexpression of HOXA-AS2 by transfecting Huh7 cell lines with pcDNA-HOXA-AS2 expression vector; F: CCK8 assay showing knockdown of HOXA-AS2 inhibited cell proliferation of HCCLM3 cells; G: CCK8 assay showing knockdown of HOXA-AS2 inhibited cell proliferation of MHCC97H cells; H: CCK8 assay showing overexpression of HOXA-AS2 promoted cell proliferation of Huh7 cells; I: Colony-formation assays showed that silencing of HOXA-AS2 significantly inhibited the colony-forming ability of HCCLM3 and MHCC97H cells, and overexpression of HOXA-AS2 significantly promoted the colony-forming ability of Huh7 cells; All tests were at least performed three times. Data were expressed as mean $\pm \mathrm{SD}$. ${ }^{* *} \mathrm{P}<0.01$.

hrs after transfection in HCCLM3 and MHCC97H cells $(P<0.01$, Fig. 2A-B). Overexpression of HOXA-AS2 increased the S-phase percentage and decreased G0/G1 phase percentage of Huh7 cells $(P<0.01$, Fig. 2C). In addition, the pro-apoptotic indexes of HOXA-AS2-silenced HCCLM3 and MHCC97H cells were obviously increased $(P<0.01$, Fig. 2D-E). As expected, the cell apoptosis was markedly decreased in Huh7 cells by pcDNA 3.1-HOXA-AS2 $(P<0.01$, Fig. 2F).

HOXA-AS2 promotes migration of HCC cell lines in vitro

The transwell assay showed that the migratory and invasive capacities of HCCLM3 and MHCC97H cells dramatically decreased after deregulation of HOXA-AS2 $(P<0.01$, Fig. 3A$B)$. Furthermore, the transwell assay showed that overexpression of HOXA-AS2 treatment significantly promoted the migration capacity of Huh7 cells compared to NC $(P<0.01$, Fig. 3C) suggesting that HOXA-AS2 could promote motility and migration of HCC cells. Because 


\section{Cellular Physiology Cell Physiol Biochem 2018:50:2124-2138

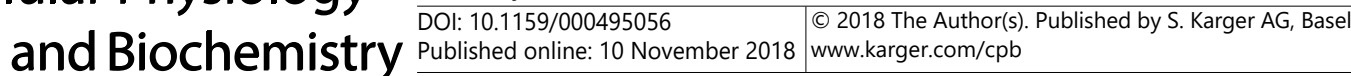 Zhang et al.: HOXA-AS2 and HCC}

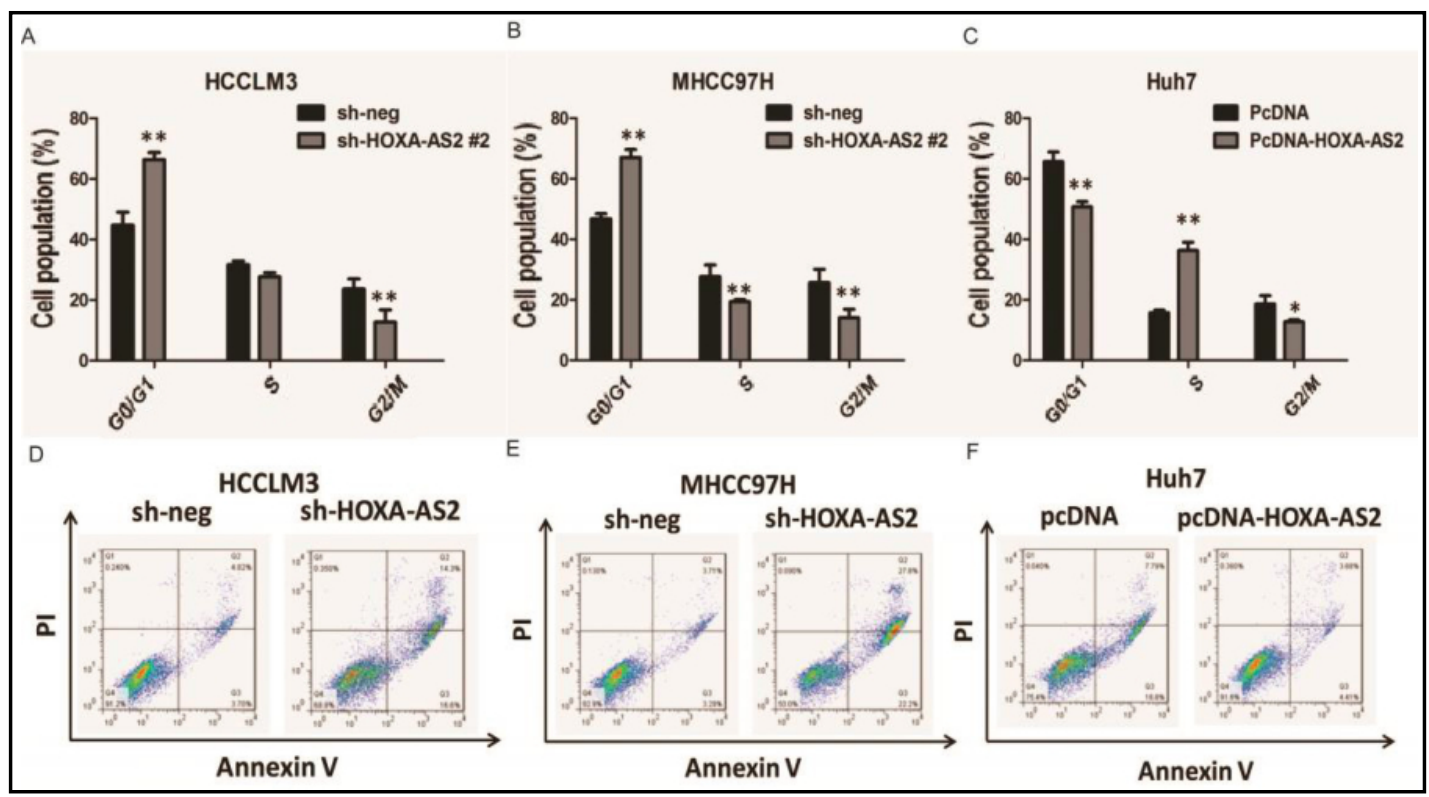

Fig. 2. A: The flow cytometry assay showed that HCCLM3 cells transfected with shHOXA-AS2 \#2 had cellcycle arrest at the G1-G0 phase in comparison with control cells; B: The flow cytometry assay showed that MHCC97H cells transfected with shHOXA-AS2 \#2 had cell-cycle arrest at the G1-G0 phase in comparison with control cells; C: The flow cytometry assay showed that Huh7 cells transfected with pcDNA 3.1-HOXAAS2 had cell-cycle arrest at the S phase in comparison with control cells; D: The flow cytometry assay showed that HCCLM3 cells transfected with shHOXA-AS2 \#2 had higher apoptotic rate in comparison with control cells; E: The flow cytometry assay showed that MHCC97H cells transfected with shHOXA-AS2 \#2 had higher apoptotic rate in comparison with control cells; F: The flow cytometry assay showed that Huh7 cells transfected with pcDNA 3.1-HOXA-AS2 had lower apoptotic rate in comparison with control cells; All tests were at least performed three times. Data were expressed as mean $\pm \mathrm{SD}$. ${ }^{*} \mathrm{P}<0.05,{ }^{* *} \mathrm{P}<0.01$.

EMT is vital for cell invasion, we next examined whether silencing HOXA-AS2 expression inhibited mesenchymal features. As expected, HOXA-AS2 knockdown decreased the expression of Vimentin and N-cadherin, and increased E-cadherin expression in HCCLM3 and MHCC97H cells (Fig. 3 D-E). Overexpression of HOXA-AS2 treatment significantly decreased the expression of E-cadherin, and increased Vimentin and N-cadherin expression in Huh7 cells (Fig. 3F). Therefore, inhibition of HOXA-AS2 in HCC cells changed the cell morphology from a mesenchymal to a more epithelial phenotype.

\section{HOXA-AS2 promotes HCC cell proliferation in vivo}

To further validate the function of HOXA-AS2, we injected HCCLM3 cells transfected with either empty vector or shHOXA-AS2 \#2 into male nude mice. Compared with the vector control, deregulation of HOXA-AS2 significantly reduced tumor growth $(P<0.01$, Fig. 4A-B). After 20 days of growth, the average tumor weight in sh-HOXA-AS2 group was significantly lower than that in the control group $(P<0.01$, Fig. 4C). The average expression level of HOXAAS2 in sh-HOXA-AS2 group was lower than that in empty group $(P<0.01$, Fig. 4D). Moreover, the suppression of Ki-67 expression of tumor tissue in the sh-HOXA-AS2 group was also observed via immunohistochemical staining $(P<0.01$, Fig. 4E-F).

HOXA-AS2 inhibited miR-520c-3p expression in HCC

To investigate the effect of HOXA-AS2 on the expression of miRNAs, HCCLM3 and MHCC97H cells were transfected with either empty vector or shHOXA-AS2 \#2 for $48 \mathrm{hrs}$. Hierarchical clustering of differentially expressed miRNAs is shown in Fig. 5A. Then, we applied the online software starBase v2.0. to predict the miRNAs that interacted with HOXAAS2. Bioinformatics analysis of miRNA recognition sequences on HOXA-AS2 revealed the 


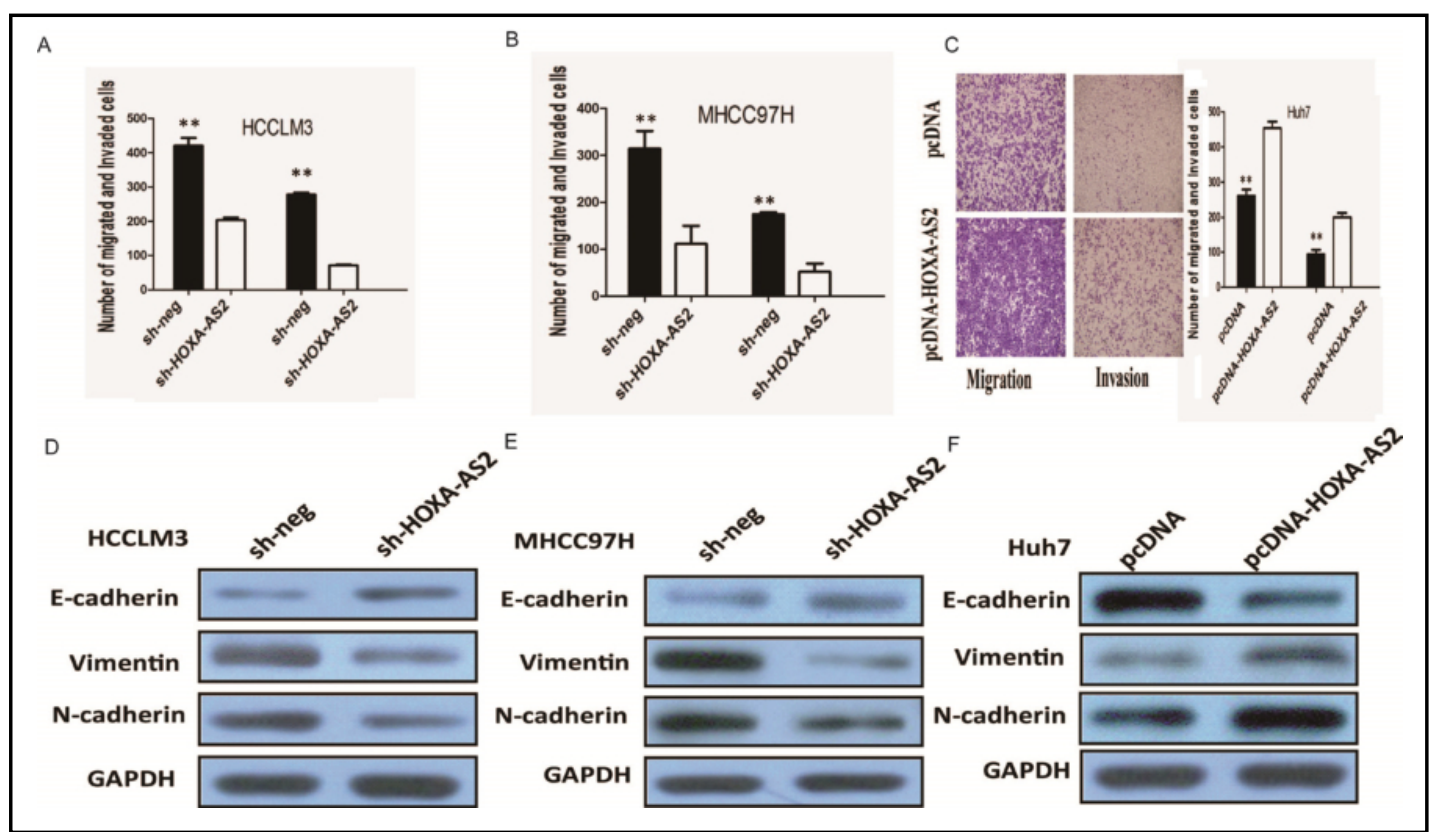

Fig. 3. A: Inhibition of migration and invasion of HCCLM3 cells transfected with shHOXA-AS2 \#2; B: Inhibition of migration and invasion of MHCC97H cells transfected with shHOXA-AS2 \#2; C: Overexpression of HOXA-AS2 treatment significantly promoted the migration capacity of Huh7 cells; D: Knockdown of HOXA-AS2 reverses EMT in HCCLM3 cells; E: Knockdown of HOXA-AS2 reverses EMT in MHCC97H cells; F: Overexpression of HOXA-AS2 promotes EMT in Huh7 cells.

Fig. 4. HOXA-AS2 knockdown inhibits HCC tumour growth in vivo. A-B, The tumour volume curve of nude mice was analysed. $\mathrm{C}$, The tumour weights of nude mice were measured. D, The expression level of HOXAAS2 in tumours of nude mice was detected by qRT-PCR. E, IHC analysis were performed to examine the expression levels of Ki-67 in tumours of nude mice. F, Knockdown of HOXAAS2 significantly decreased the percentage of Ki-67 positive cells in tumours of nude mice. ${ }^{*} \mathrm{P}<0.05,{ }^{* *} \mathrm{P}<0.01$.

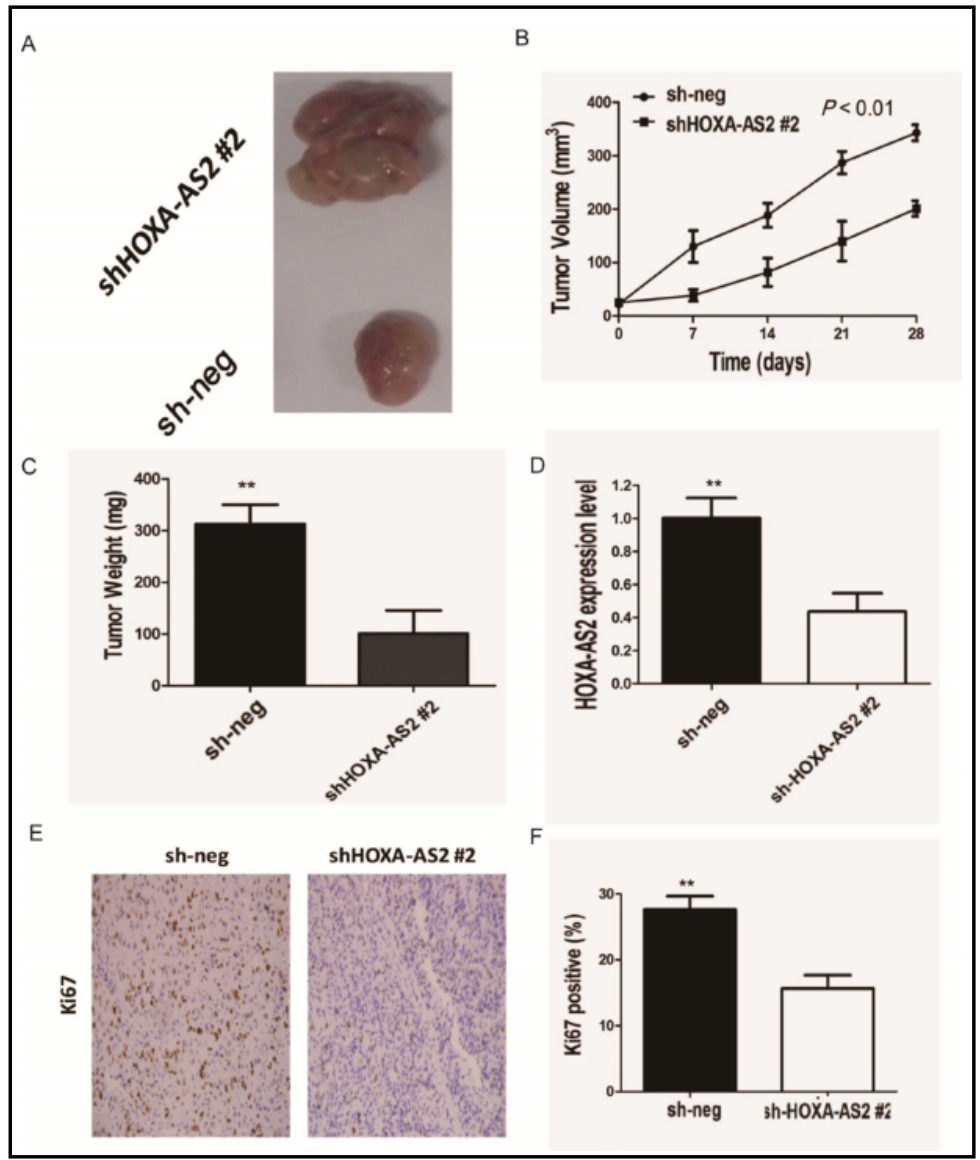




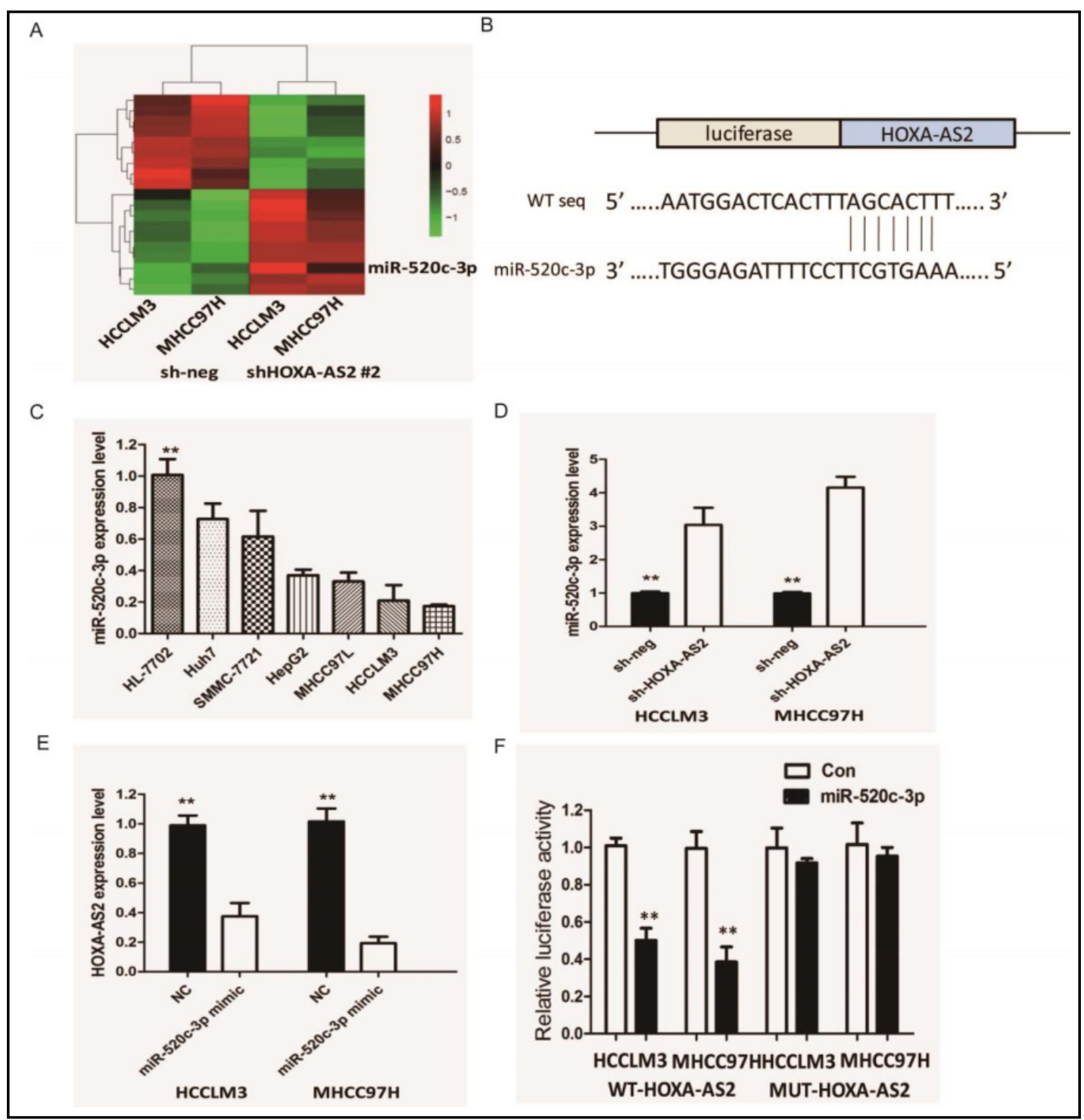

Fig. 5. A: HCCLM3 and MHCC97H cells were transfected with shHOXA-AS2 \#2 for 48 hrs. Hierarchical clustering revealed systematic variations in the expression of miRNAs. Numerous differentially expressed miRNAs between control and shHOXA-AS2 \#2 transfected U2HCC cells are shown on a scale from green (low) to red (high); B: StarBase v2.0 results showing the sequence of HOXA-AS2 with highly conserved putative miR-520c-3p binding sites; C: Expression levels of miR-520c-3p in different HCC cell lines were determined by qRT-PCR; D: Silencing of HOXA-AS2 increased the expression level of miR-520c-3p in HCCLM3 and MHCC97H cells; E: miR-520c-3p inhibited the expression of HOXA-AS2 in HCCLM3 and MHCC97H cells; F: The wild-type or mutant miR-520c-3p-binding sites in HOXA-AS2 were inserted into pMIR-report luciferase vector. Luciferase activity was detected in HCC cells co-transfected with miR-520c-3p or negative control (miR-control) and reporter plasmids containing WT-HOXA-AS2 (wild type) or MUT-HOXA-AS2 (mutant type). The normalized luciferase activity in the miR-control group was used as the relative luciferase activity. All tests were at least performed three times. Data were expressed as mean $\pm \mathrm{SD}$. ${ }^{*} \mathrm{P}<0.05,{ }^{* *} \mathrm{P}<0.01$.

presence of miR-520c-3p binding sites (Fig. 5B). Next, we measured the levels of miR-520c$3 p$ expression in various HCC cell lines. As shown in Fig. 5C, the expression of miR-520c-3p was obviously decreased in HCCLM3 and MHCC97H cells, indicating the opposite result to HOXA-AS2 expression. 
Fig. 6. A: qRT-PCR showing expression level of miR-520c-3p in HCC tissues and adjacent noncancerous tissues; B: Pearson's correlation analysis revealed HOXA-AS2 and miR-520c-3p expressions were inversely correlated in HCC tissues; C: miR-520c-3p mimic was used to force the expression of miR520c-3p in HCCLM3 cells; D: miR-520c-3p mimic was used to force the expression of miR-520c-3p in MHCC97H cells; E: $\quad$ miR-520c-3p inhibitor was used to knockdown the expression of miR520c-3p in Huh7 cells; F: CCK8 assay showing miR-520c$3 p$ mimic inhibited cell proliferation of HCCLM3 cells; G: CCK8 assay showing miR-520c$3 p$ mimic inhibited cell proliferation of MHCC97H cells; $\mathrm{H}$ : CCK8 assay showing miR-520c-3p inhibitor promoted cell proliferation of Huh7

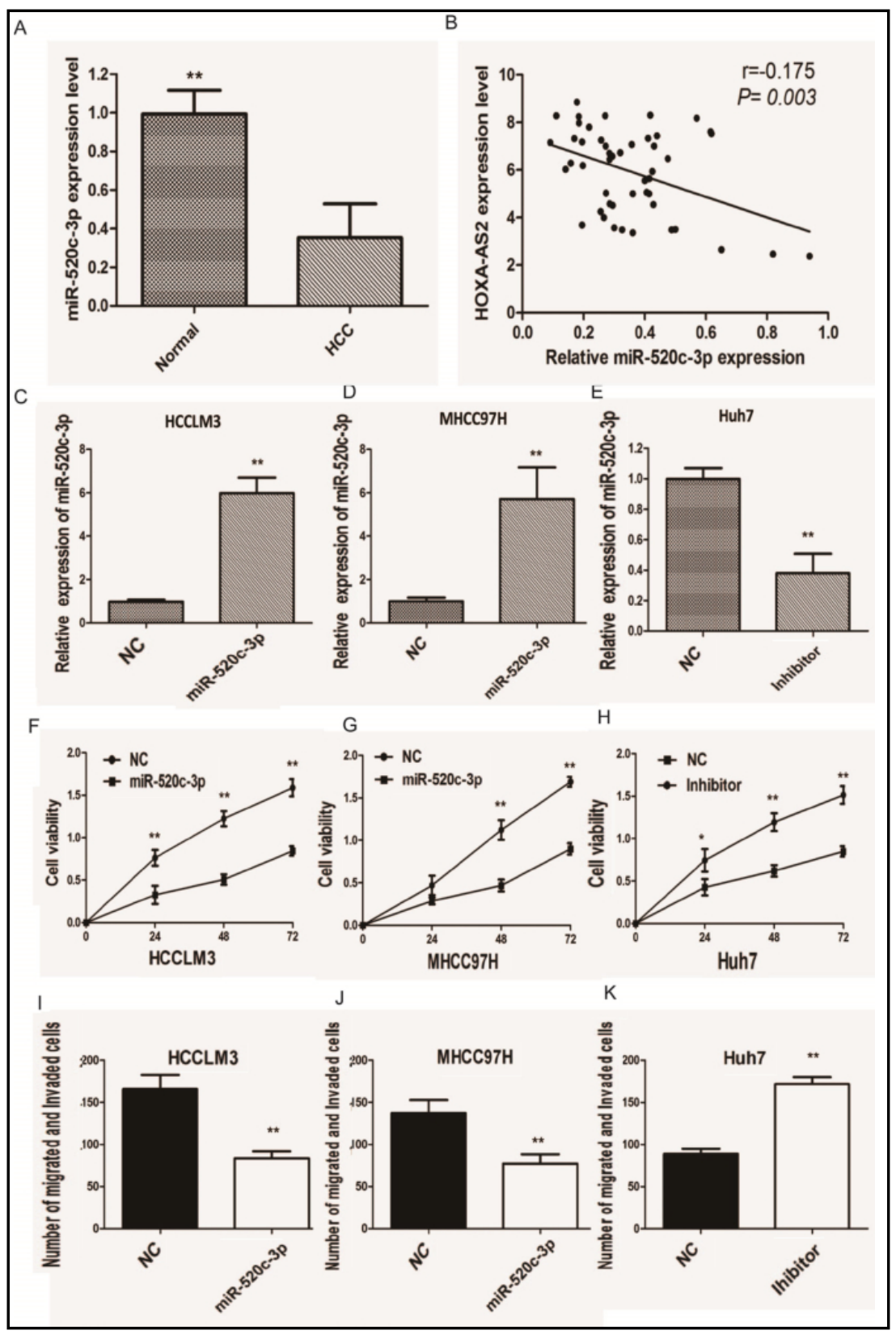
cells; I: Transwell assay showing miR-520c-3p mimic inhibited cell invasion of HCCLM3 cells; J: Transwell assay showing miR520c-3p mimic inhibited cell invasion of MHCC97H cells; K: Transwell assay showing miR-520c-3p inhibitor promoted cell invasion of invasion of Huh7 cells; All tests were at least performed three times. Data were expressed as mean $\pm \mathrm{SD}$. ${ }^{* *} \mathrm{P}<0.01$.

To explore the regulatory relationship between HOXA-AS2 and miR-520c-3p, HCCLM3 and MHCC97H cells were transfected with either empty vector or shHOXA-AS2 \#2, and qRTPCR was used to detect the expression level of miR-520c-3p. The results showed that silencing of HOXA-AS2 increased the expression level of miR-520c-3p in HCCLM3 and MHCC97H cells $(P<0.01$, Fig. 5D). Meanwhile, miR-520c-3p mimics also inhibited the expression of HOXAAS2 in HCCLM3 and MHCC97H cells $(P<0.01$, Fig. 5E). 


\section{Cellular Physiology Cell Physiol Biochem 2018;50:2124-2138 \begin{tabular}{ll|l} 
and Biochemistry & $\begin{array}{l}\text { DOl: 10.1159/000495056 } \\
\text { Published online: 10 November } 2018\end{array}$ & $\begin{array}{l}\text { O } 2018 \text { The Author(s). Published by S. Karger AG, Basel } \\
\text { www.karger.com/cpb }\end{array}$ \\
\cline { 2 - 3 }
\end{tabular} \\ Zhang et al.: HOXA-AS2 and HCC}

For further confirmation, we used the luciferase assay to detect the direct binding between HOXA-AS2 and miR-520c-3p. We cloned the wild type sequence of HOXA-AS2 or its mutant sequence into the pMIR luciferase reporter, and co-transfected the reporter plasmid (or the corresponding mutant reporter) and miR-520c-3p mimics (or negative control) into HCCLM3 and MHCC97H cells, respectively. The results showed that miR-520c-3p overexpression considerably reduced the luciferase activity of the HOXA-AS2-wt luciferase reporter vector compared with negative control, while miR-520c-3p overexpression did not pose any impact on the luciferase activity of HOXA-AS2-mt-transfected cells $(P<0.01$, Fig. $5 F)$. All these data demonstrated that HOXA-AS2 associated with the miR-520c-3p and may function as a ceRNA.

miR-520c-3p inhibits HCC cell proliferation and invasion by targeting GPC3

Then, we detected the levels of miR-520c-3p in 58 pairs of HCC tissues together with adjacent non-tumour tissues by the qRT-PCR assay. It was revealed by our findings that miR$520 c-3 p$ expression was considerably downregulated in HCC tissues, in comparison with the adjacent non-tumour tissuess $(P<0.01$; Fig. 6A), and miR-520c-3p expression was inversely correlated with HOXA-AS2 expression in HCC tissues (R=-0.175, $P=0.003$, Fig. 6B). In addition, the relationships between miR-520c-3p expression and clinical characteristics of HCC patients were analyzed, and we found that lower miR-520c-3p expression was significantly associated with distant metastasis $(P=0.0012)$ and TNM stage $(P<0.0001)$, which has a negative relationship with HOXA-AS2. To validate whether miR-520c-3p could also inhibit HCC cell proliferation and invasion, we used the mimic treatment to force the expression of miR-520c-3p in HCCLM3 and MHCC97H cells, whereas knockdown the miR-520c-3p level by treatment with miR-520c-3p inhibitor in Huh7 cells $(P<0.01$; Fig. 6C-E). Functionally, the results of the CCK8 and transwell assays revealed that miR-520c-3p overexpression markedly inhibits HCCLM3 and MHCC97H cell growth and invasion, which is consistent with the results of HOXA-AS2 expression knockdown, whereas miR-520c-3p knockdown by specific inhibitor promotes cell proliferation and invasion in Huh7 cells $(P<0.01$; Fig. 6F-K).

To identify potential target genes of miR-520c-3p, we searched for candidate genes using TargetScan6.2 and miRBase miRNA databases. Bioinformatics analysis showed that miR-520c-3p directly targets GPC3 (Fig. 7A). To confirm the predictions, a wild type or mutant GPC3 3' UTR luciferase reporter vector was conducted. GPC3-wt or GPC3-mut was cotransfected with miR-520c-3p mimics or negative control into HEK293T cells. The relative luciferase activity was remarkably reduced in cells co-transfected with the GPC3wt luciferase reporter and miR-520c-3p mimic than in the negative control cells. However, inhibitory effects were abolished when $3^{\prime}$-UTRs that contained both mutant-binding sites were co-transfected with miR-520c-3p, confirming that GPC3 is a target of miR-520c-3p $(P<0.01$; Fig. 7B $)$.

Furthermore, qRT-PCR analysis revealed that transfection of miR-520c-3p mimics in HCCLM3 and MHCC97H cells led to a considerably reduction of GPC3 expression at mRNA levels $(P<0.01 ;$ Fig. $7 \mathrm{C})$. Then, the qRT-PCR was performed to detect the expression of GPC3 mRNA in 58 paired HCC tissues and corresponding nonneoplastic liver tissues. GPC3 expression was significantly higher in HCC tissues compared with the adjacent nontumoral liver tissue $(P<0.01$; Fig. 7D). The immunohistochemistry results showed that GPC3 expression in HCC specimens was significantly upregulated compare with that in the adjacent non-tumoral liver tissue (41/58 VS. 18/58, $P<0.0001$; Fig. 7E). Thereafter, the Pearson's correlation analysis indicated that miR-520c-3p expression level had inverse correlation with the expression of GPC3 mRNA in HCC tissues. These evidences demonstrated that GPC3 was a direct target of miR-520c-3p in HCC. 


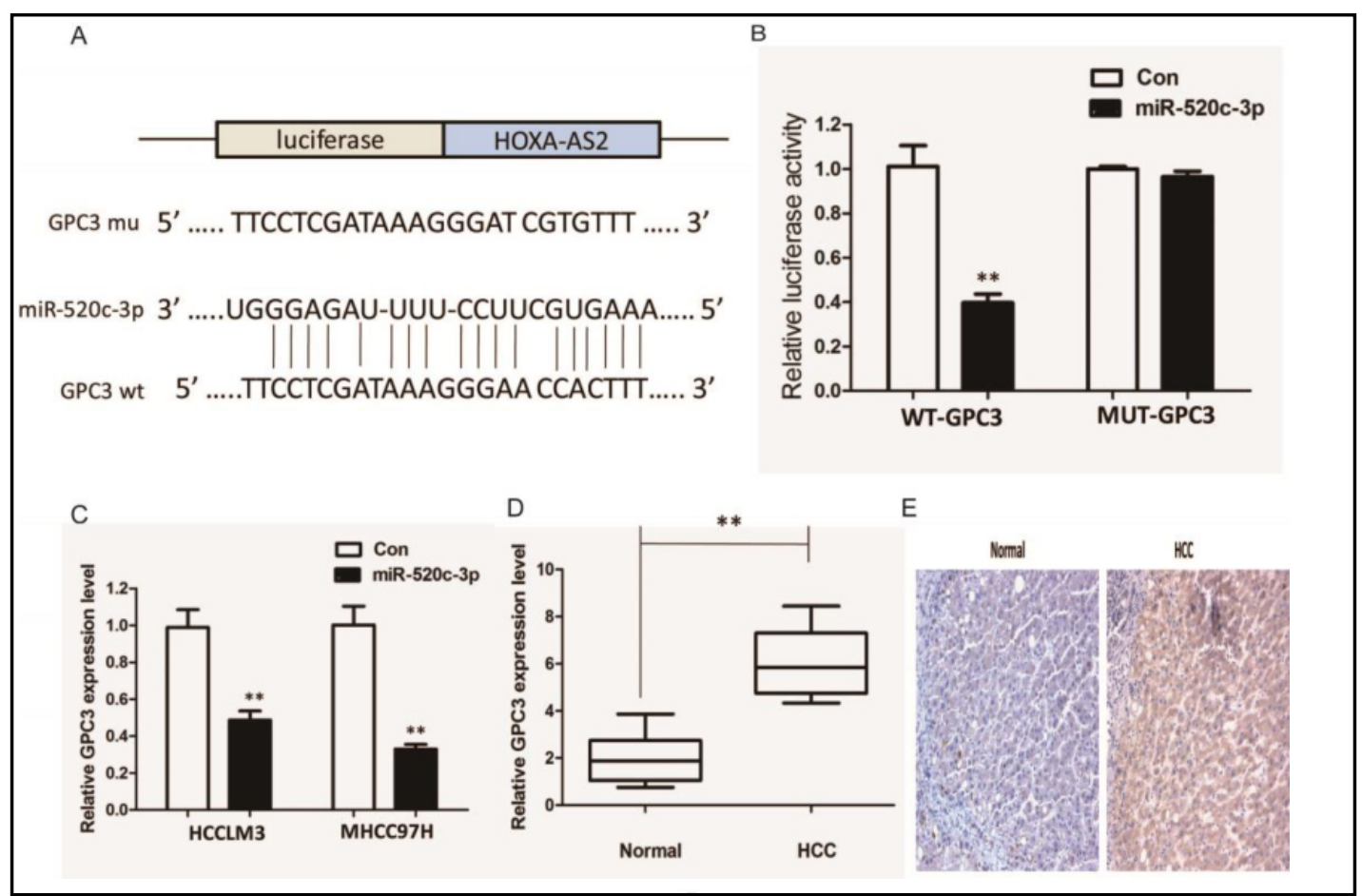

Fig. 7. GPC3 is a direct target of miR-520c-3p in HCC. A: Bioinformatics analysis revealed the predicted binding sites between GPC3 and miR-520c-3p. B: Luciferase reporter assay demonstrated miR-520c3p mimics significantly decreased the luciferase activity of GPC3-wt in HEK293T cells. C: miR-520c-3p overexpression in HCCLM3 and MHCC97H cells decreased the mRNA expression of GPC3 compared to negative control. D: GPC3 expression was significantly upregulated in HCC tissues compared with adjacent normal tissues by qRT-PCR analysis; E: IHC analysis were performed to examine the expression levels of GPC3 in HCC tissues and adjacent normal tissues; ${ }^{* *} \mathrm{P}<0.01$.

HOXA-AS2 controls the miR-520c-3p target, GPC3

HOXA-AS2 directly binds miR-520c-3p and inhibits its activity, and miR-520c-3p targets GPC3. We therefore speculated whether HOXA-AS2 controls GPC3 expression in a miR-520c3p-dependent manner. Expectedly, forced expression of HOXA-AS2 significantly upregulated endogenous GPC3 mRNA expression in Huh7 cells. Furthermore, forced expression of miR$520 c-3 p$ abrogates HOXA-AS2-inducing upregulation of GPC3 $(P<0.01$; Fig. 8A). Inversely, knockdown of HOXA-AS2 decreases GPC3 expression level in HCCLM3 and MHCC97H cells. For the rescue experiment, we inhibited miR-520c-3p by the inhibitor in HOXA-AS2downregulated HCC cells $(P<0.01$; Fig. 8B-C).

To further verify whether this observed effect depends on the role miR-520c-3p in regulating of the GPC3, 3'UTR, luciferase activity was assayed after cotransfection of luciferase plasmid (GPC3-3'UTR) with HOXA-AS2. Overexpression of HOXA-AS2, but not the mutant, increases the relative luciferase activity, whereas forced expression of miR-520c-3p abolishes this increase $(P<0.01$; Fig. 8D). HOXA-AS2 level is positively associated with GPC3 mRNA level. These results suggest that HOXA-AS2 positively regulates GPC3 expression by competitively binding miR-520c-3p. 


\begin{abstract}
Discussion
In this study, we
have demonstrated that upregulation of HOXA-AS2 in HCC could be an important contributor to its increased growth, and enhancive migration and invasion. We found that HOXA-AS2 was highly expressed in HCC and metastatic HCC tissues and HCC cell lines, while miR520c-3p expression was low in HCC tissues and HCC cell lines. Moreover, down-regulation of HOXA-AS2 inhibited the proliferation, migration,
\end{abstract} invasion of HCCs in vitro and in vivo. Specifically, we also showed mechanistically that HOXA-AS2 promotes the progression of HCC by inhibiting miR-520c-3p expression. Furthermore, evidence for HOXA-AS2 functionality in HCC also extended to clinicopathological features of HCC patients, and may be considered for the development of potential therapeutics against HCC.

It is becoming evident that IncRNAs have been demonstrated to play an important in various human pathophysiologic processes, especially carcinogenesis [18-20]. A number of recent papers have revealed that HCC has also been shown to have differential lncRNA expressions [21, 22]. HOXA-AS2 was first identified in all-trans retinoic acid-treated NB4 promyelocytic leukemia cells, and the functional role of HOXA-AS2 was demonstrated in various types of cancers [23, 24]. Lian et al. demonstrated that IncRNA-HOXA-AS2/EZH2/LSD1 complex may function as an oncogene in pancreatic cancer cell proliferation, and also provides a potential therapy target for pancreatic cancer [25]. Gao et al. found that HOXA-AS2 knockdown inhibited malignant glioma behaviors and VM formation via the miR-373/EGFR axis [26]. However, the expression and biological function of HOXA-AS2 in HCC are poorly understood. HOXAAS2 was found to be up-regulated in the HCC tissues and HOXA-AS2 was associated with poor prognosis in HCC patients. In agreement with previous studies, we demonstrated that HOXAAS2 was significantly upregulated in the HCC tissues compared with the normal liver tissues. In addition, increased expression of the HOXA-AS2 is associated with distant metastasis and advanced TNM stage. in vitro functional assays showed that knockdown of HOXA-AS2 significantly inhibited cell proliferation, invasion and migration, while overexpression of HOXA-AS2 promoted cell proliferation, invasion and migration in HCC cell lines. In addition, HOXA-AS2 knockdown promoted significant arrest in the G0/G1-phase and resulted in an obvious increase of apoptosis in HCC cell line, whereas overexpression has the opposite 


\section{Cellular Physiology Cell Physiol Biochem 2018;50:2124-2138

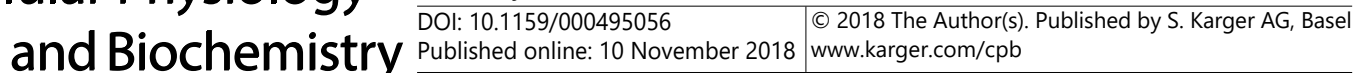 \\ Zhang et al.: HOXA-AS2 and HCC}

results, suggesting that HOXA-AS2 may play an oncogenic role in HCC. Several oncogenic cues have been suggested could increase HCC cell growth and stemness through promoting EMT process [27]. Our results showed that HOXA-AS2 impairs cell invasion and metastasis through the regulation of EMT process. HOXA-AS2 can certainly increase the expression level of vimentin and $\mathrm{N}$-cadherin, and inhibit E-Cadherin expression.

LncRNAs are potentially implicated in determining cancer development through binding with miRNAs that have a wide range of targets [26]. So we performed a microarray to screen the HOXA-AS2-regulated miRNAs in HCC. Bioinformatics analysis and luciferase assays were also used to validate the direct binding ability of the predicted miRNA response elements on the full-length HOXA-AS2 transcript. We identified miR-520c-3p as a potential target of HOXA-AS2. An observation for the existence of an inverse correlation between HOXA-AS2 and miR-520c-3p expressions in HCC tissues was made. More importantly, the gain and loss function assay indicated that HOXA-AS2 can suppress the expression of miR-520c-3p, and this interaction has a reciprocal effect. We also found that miR-520c-3p was a tumor suppressor gene in HCC by suppressing cell proliferation and invasion, which was consistent with results of knockdown of HOXA-AS2 expression in HCC cells.

In the present study, GPC3 was predicted as a downstream target of miR-520c-3p, which was confirmed by luciferase reporter assay. We found that GPC3 was up-regulated in HCC tissues and the mRNA expression level of HPC3 was negatively correlated with miR-520c$3 p$ expression, but positively correlated with HOXA-AS2 expression in HCC tissues. The luciferase assay confirmed the existence of specific crosstalk between HOXA-AS2 and GPC3 mRNA through competition for miR-520c-3p binding.

\section{Conclusion}

In conclusion, our study identified HOXA-AS2 as an oncogene that performed a pivotal function in cell proliferation and metastasis of HCC. Furthermore, HOXA-AS2 could positively regulate GPC3 expression through modulation of miR-520c-3p in HCC cells. The HOXA-AS2/ miR-520c-3p/GPC3 regulatory network may shed light on tumorigenesis in HCC and may contribute to the development of new efficient therapeutic approaches for HCC.

\section{Disclosure Statement}

The authors declare that they have no competing interests.

\section{References}

1 Yang JD, Roberts LR: Hepatocellular carcinoma: A global view. Nat Rev Gastroenterol Hepatol 2010;7:448458.

-2 El-Serag HB, Marrero JA, Rudolph L, Reddy KR: Diagnosis and treatment of hepatocellular carcinoma. Gastroenterology 2008;134:1752-1763.

-3 El-Serag HB: Hepatocellular carcinoma. N Engl J Med 2011;365:1118-1127.

4 Harlan LC, Parsons HM, Wiggins CL, Stevens JL, Patt YZ: Treatment of hepatocellular carcinoma in the community: disparities in standard therapy. Liver Cancer 2015;4:70-83.

5 Kornienko AE, Guenzl PM, Barlow DP, Pauler FM: Gene regulation by the act of long non-coding RNA transcription. BMC Biol 2013;11:59.

6 Ponting CP, Oliver PL, Reik W: Evolution and functions of long noncoding RNAs. Cell 2009;136:629-641.

7 Xu XF, Li J, Cao YX, Chen DW, Zhang ZG, He XJ, Ji DM, Chen BL: Differential Expression of Long Noncoding RNAs in Human Cumulus Cells Related to Embryo Developmental Potential: A Microarray Analysis. Reprod Sci 2015;22:672-678. 


\section{Cellular Physiology Cell Physiol Biochem 2018;50:2124-2138

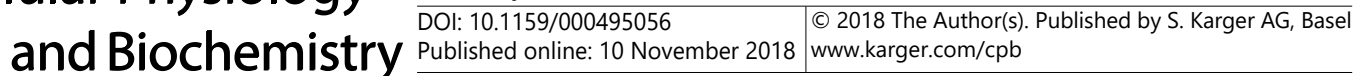

8 Chalei V, Sansom SN, Kong L, Lee S, Montiel JF, Vance KW, Ponting CP: The long non-coding RNA Dali is an epigenetic regulator of neural differentiation. Elife 2014;3:e04530.

-9 Braconi C, Kogure T, Valeri N, Huang N, Nuovo G, Costinean S, Negrini M, Miotto E, Croce CM, Patel T: microRNA-29 can regulate expression of the long non-coding RNA gene MEG3 in hepatocellular cancer. Oncogene 2011;30:4750-4756.

10 He JH, Han ZP, Liu JM, Zhou JB, Zou MX, Lv YB, Li YG, Cao MR: Overexpression of Long Non-Coding RNA MEG3 Inhibits Proliferation of Hepatocellular Carcinoma Huh7 Cells via Negative Modulation of miRNA-664. J Cell Biochem 2017;118:3713-3721.

11 Zhao H, Zhang X, Frazao JB, Condino-Neto A, Newburger PE: HOX antisense lincRNA HOXA-AS2 is an apoptosis repressor in all trans retinoic acid treated NB4 promyelocytic leukemia cells. J Cell Biochem 2013;114:2375-2383.

12 Salmena L, Poliseno L, Tay Y, Kats L, Pandolfi PP: A ceRNA hypothesis: the Rosetta Stone of a hidden RNA language? Cell 2011;146:353-358.

13 McCarthy N: Regulatory RNA: layer by layer. Nat Rev Genet 2011;12:804.

14 Carthew RW, Sontheimer EJ: Origins and Mechanisms of miRNAs and siRNAs. Cell 2009;136:642-655.

-15 Fang Y, Wang J, Wu F, Song Y, Zhao S, Zhang Q: Long non-coding RNA HOXA-AS2 promotes proliferation and invasion of breast cancer by acting as a miR-520c-3p sponge. Oncotarget 2017;8:46090-46103.

-16 Tang CP, Zhou HJ, Qin J, Luo Y, Zhang T: MicroRNA-520c-3p negatively regulates EMT by targeting IL-8 to suppress the invasion and migration of breast cancer. Oncol Rep 2017;38:3144-3152.

17 Mudduluru G, Ilm K, Fuchs S, Stein U: Epigenetic silencing of miR-520c leads to induced S100A4 expression and its mediated colorectal cancer progression. Oncotarget 2017;8:21081-21094.

18 Gao P, Wei GH: Genomic Insight into the Role of IncRNA in Cancer Susceptibility. Int J Mol Sci 2017;18.

19 Peng WX, Koirala P, Mo YY: LncRNA-mediated regulation of cell signaling in cancer. Oncogene 2017;36:5661-5667.

-20 Graham LD, Pedersen SK, Brown GS, Ho T, Kassir Z, Moynihan AT, Vizgoft EK, Dunne R, Pimlott L, Young GP, Lapointe LC, Molloy PL: Colorectal Neoplasia Differentially Expressed (CRNDE), a Novel Gene with Elevated Expression in Colorectal Adenomas and Adenocarcinomas. Genes Cancer 2011;2:829-840.

-21 Lv H, Lv G, Han Q, Yang W, Wang H: Noncoding RNAs in liver cancer stem cells: The big impact of little things. Cancer Lett 2018;418:51-63.

-22 Liu Y, Yang Y, Wang T, Wang L, Wang X, Li T, Shi Y, Wang Y: Long non-coding RNA CCAL promotes hepatocellular carcinoma progression by regulating AP-2alpha and Wnt/beta-catenin pathway. Int J Biol Macromol 2018;109:424-434.

-23 Wang F, Yang H, Deng Z, Su Y, Fang Q, Yin Z: HOX Antisense lincRNA HOXA-AS2 Promotes Tumorigenesis of Hepatocellular Carcinoma. Cell Physiol Biochem 2016;40:287-296.

24 Xie M, Sun M, Zhu YN, Xia R, Liu YW, Ding J, Ma HW, He XZ, Zhang ZH, Liu ZJ, Liu XH, De W: Long noncoding RNA HOXA-AS2 promotes gastric cancer proliferation by epigenetically silencing P21/PLK3/DDIT3 expression. Oncotarget 2015;6:33587-33601.

-25 Lian Y, Li Z, Fan Y, Huang Q, Chen J, Liu W, Xiao C, Xu H: The lncRNA-HOXA-AS2/EZH2/LSD1 oncogene complex promotes cell proliferation in pancreatic cancer. Am J Transl Res 2017;9:5496-5506.

-26 Gao Y, Yu H, Liu Y, Liu X, Zheng J, Ma J, Gong W, Chen J, Zhao L, Tian Y, Xue Y: Long Non-Coding RNA HOXAAS2 Regulates Malignant Glioma Behaviors and Vasculogenic Mimicry Formation via the MiR-373/EGFR Axis. Cell Physiol Biochem 2018;45:131-147.

-27 Zhang LG, Zhou XK, Zhou RJ, Lv HZ, Li WP: Long non-coding RNA LINC00673 promotes hepatocellular carcinoma progression and metastasis through negatively regulating miR-205. Am J Cancer Res 2017;7:2536-2544. 